\title{
Interactive Dictionary for Visually Impaired
}

\author{
Reena Atul Panchal ${ }^{1}$, Rachana Papewar ${ }^{2}$, Chaitali Gawli ${ }^{3}$, Khushboo \\ Hotchandani ${ }^{4}$
}

Department Of Electronics and Telecommunication Watumull Institute of Electronic Engineering and Computer Technology Worli, Mumbai-18,India.

\begin{abstract}
There is a growing awareness among parents, teachers, blind youth, and the adult blind community that the education which blind children are receiving is failing them. They are not learning to use and trust the alternative techniques which blind persons must have if they are to be successful.

In this paper we are presenting interactive learning tools for visually impaired people. It is an innovative technique that helps the blind people to overcome the problems which they face during their educational training. It is an audio dictionary which gives synonyms of words spoken by the user through microphone. It is step ahead of the speech synthesis technology where the speech is not only synthesized but also gives the synonyms of the words in the form of audio output. We aim at developing a dictionary of up to 60 words. Mobile applications will also increase the scope. For other physically disabled people it can be further modified.
\end{abstract}

Keywords: - Visually impaired, interactive learning, audio dictionary, synonyms, speech synthesis.

\section{INTRODUCTION}

Blind people confront a number of visual challenges every day - from reading the label on a frozen dinner to figuring out if they're at the right bus stop. While many tools have been introduced to help address these problems using computer vision and other sensors (talking OCR, GPS, radar canes, etc.), their capabilities are dictated as much by the state-of-the-art in technology as they are by real human problems. A deeper understanding of the questions that blind people would like to ask in their day-to-day lives may help to direct innovation to solve them.

The lack of quality field placements for students in training is a truly serious problem. It dooms these students to go into the classroom and deliver the same wretched educational services that blind children are now receiving. Their lives are characterized by unemployment, poor housing, inadequate health care, barriers to lifelong learning, culture, sports and recreation. It would be no exaggeration to say that visually impaired persons are among the poorest people in the society which leads them to suffer oppression, marginalization and exclusion. Addressing the challenges they face requires giving attention to their educational programs by providing the needed resources and trained personnel. Our project is an attempt to provide an educational aid to the visually impaired which consists of an interactive dictionary helping them be independent and learn things.

\section{ACTUAL SYSTEM}

Braille introduced in 1829 is a tactile writing system used by the blind and the visually impaired. It is traditionally written with embossed paper. Braille-users can read computer screens and other electronic supports. They can write Braille with the original slate and stylus or type it on a Braille writer, such as a portable Braille note-taker, or on a computer that prints with a Braille embosser.

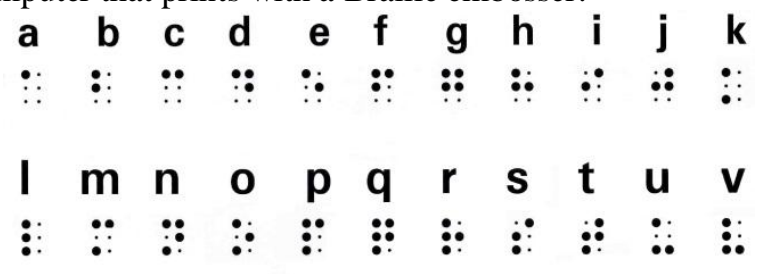

$$
\begin{array}{cccc}
\text { w } & \text { x } & \text { y } & \text { z } \\
:: & :: & :: & \text { :: }
\end{array}
$$

Figure 1: Braille Code 
JAWS (Job Access With Speech) is a computer screen reader program for Microsoft Windows that allows blind and visually impaired users to read the screen either with a text-to-speech output or by a Refreshable Braille display. It converts your computer into a talking computer. It reads out all the matter that is on the computer's screen through your computer's speakers/ headphones, thus enabling a visually challenged person to use the computer independently and work on all MS Windows applications.

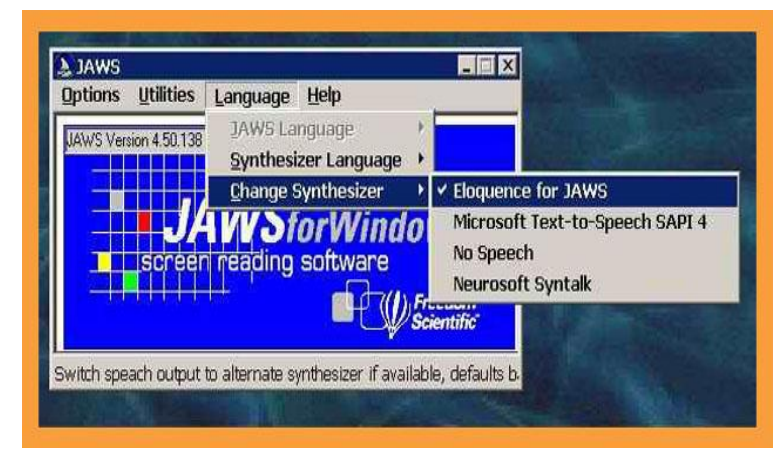

Figure 2: JAWS screen reader software

However, our project is an attempt to provide a portable interactive dictionary which gives meanings of the words spoken by the person. A complete software-based solution is attractive for a desktop application, but fails to provide an embedded portable and integrated solution. [7]

\section{PROPOSED SYSTEM}

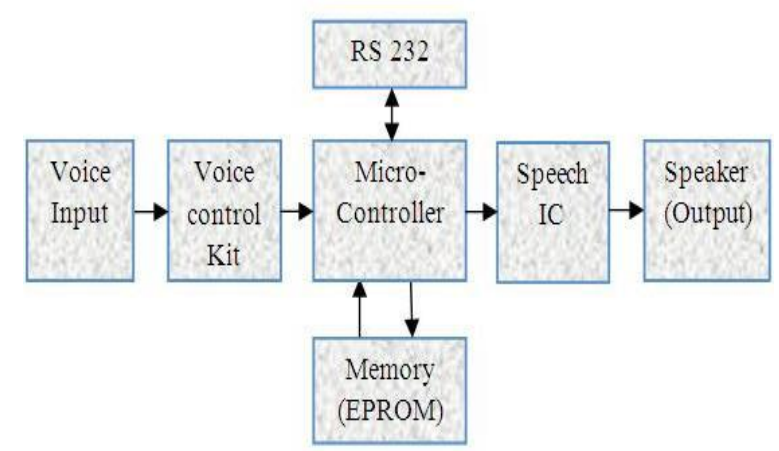

Figure 3: Basic Block Diagram

The input speech is given through the microphone which then goes to the voice control kit. This kit processes the input and the word which was spoken is recognized. This word is forwarded to the EPROM database. The database is searched for the synonym of the word spoken by the user and the synonym is given out in the speech form through the speaker.

\section{IMPLEMENTATION}

\section{A. Voice Control Kit}

The speech recognition system is a completely assembled and easy to use programmable speech recognition circuit. It has 8 bit data out which can be interfaced with any microcontroller for further development. The HM2007 is a CMOS voice recognition LSI (Large Scale Integration) circuit. The input speech first goes to the speech IC (HM2007) of the voice control kit. This IC works in two modes: [7]

- Training: Stores the database.

- Recognition: Compares with the database.

The IC requires an external memory which is sufficed by an SRAM. Speech IC along with the static RAM forms the fundamental block of the kit. The database is stored in the SRAM and then HM2007 is used in the recognition mode. As mentioned above, in recognition mode, comparison of the input and the database takes place and a particular eight bit BCD address is given as the result. This address gives the location of the found word or it represents the exception which takes place. The address remains on the D-bus of the speech IC for a 
very small time which may prove to be insufficient for extracting all the eight bits of the address. Thus, a latch is used which increases the time to read the address.

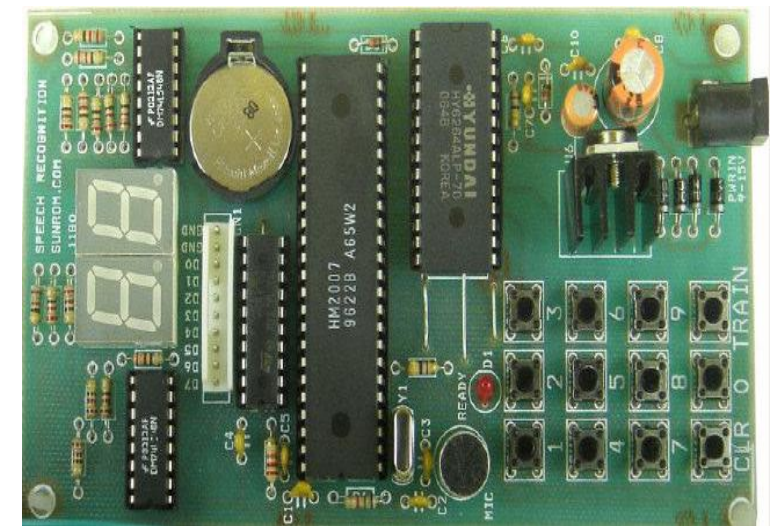

Figure 4: Image of a Voice control Kit

\section{Training Words for Recognition:}

Press "1" (display will show "01" and the LED will turn off) on the keypad, then press the TRAIN key (the LED will turn on) to place circuit in training mode, for word one. Say the target word into the onboard microphone (near LED) clearly. The circuit signals acceptance of the voice input by blinking the LED off then on. The word (or utterance) is now identified as the " 01 " word. If the LED did not flash, start over by pressing " 1 " and then "TRAIN" key. You may continue training new words in the circuit. Press "2" then TRN to train the second word and so on. The circuit will accept and recognize up to 20 words (numbers 1 through 20). It is not necessary to train all word spaces.

\section{Testing Recognition:}

Repeat a trained word into the microphone. The number of the word should be displayed on the digital display. For instance, if the word "directory" was trained as word number 20, saying the word "directory" into the microphone will cause the number 20 to be displayed.[7]

\section{Error Codes:}

The chip provides the following error codes.

$55=$ word to long

$66=$ word to short

$77=$ no match

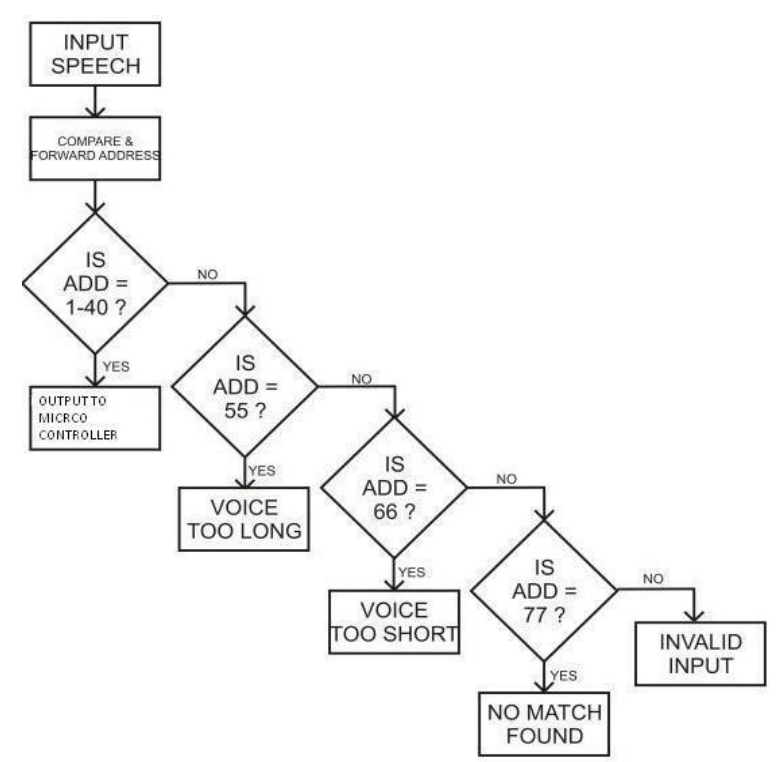

Flowchart 1: Flow of data in Voice Control Kit 
The address is forwarded to the micro-controller 8051. The micro controller searches the spoken word in the EPROM memory and the synonym of that word is forwarded to the speech IC for conversion into analog voice output.

\section{B. Speech IC SC040B}

SC040B is a $40 \mathrm{sec}$ speech chip. It supports PWM or DAC voice output with a high quality speech. It provides a 40 second voice length under $6 \mathrm{KHz}$ sampling and 4 bit hardware compression. It has an internal built-in oscillator for system clock. The IC can work in three modes as required by the user. [8]

The speech IC therefore generates analog voice of the data forwarded by the micro controller i.e. the synonym of the input word is said by the speaker.[8]

\section{METHODOLOGY}

The following flowchart explains the flow of data in the system.

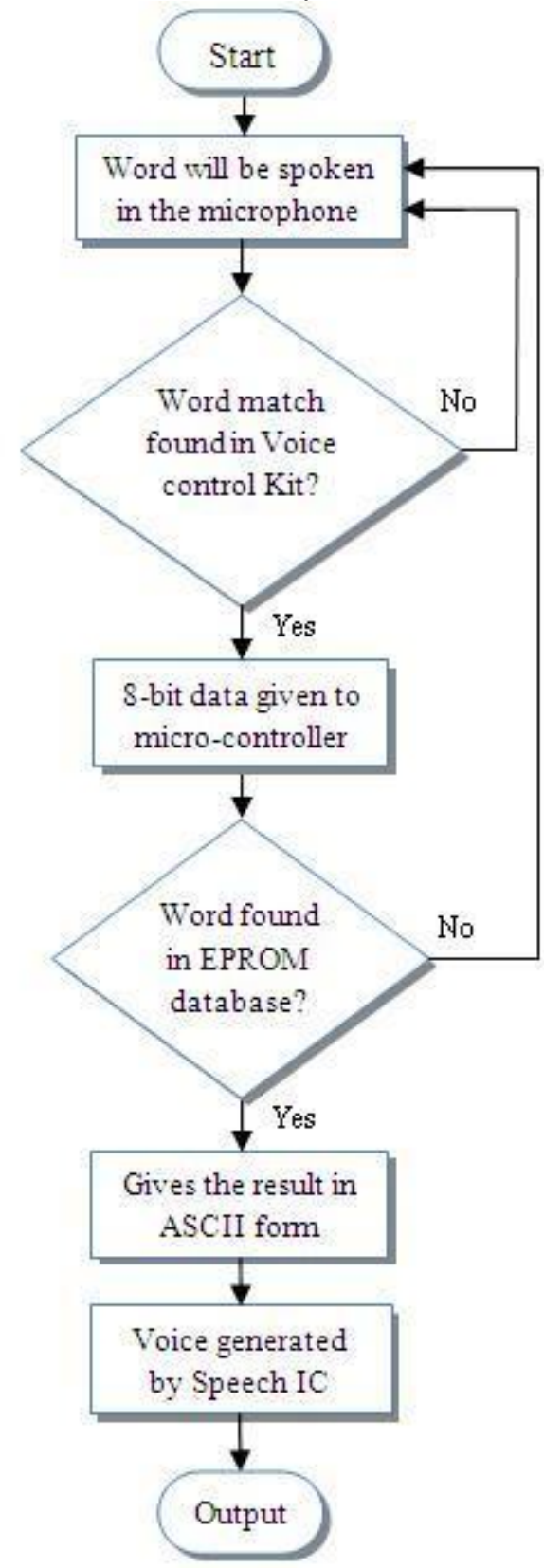

Flowchart 2: Work flow of the system

We will now understand the working with the help of an example. Suppose the blind person say a word "amplifier" in the microphone of the Voice Control Kit. This word will be searched in the RAM of the trained 
kit. If the word is found at suppose location 02 then 02 will be displayed on the seven segment display and BCD equivalent of 02 is forwarded to the micro controller. If the spoken word is not found then the whole process starts again.

The micro controller then searches for the word in the EPROM database. The synonym of the word that is stored in the database is given to the speech IC. The speech IC generates analog voice. Thus the blind person can hear the synonym of the word spoken by him from the speaker.

\section{RESULTS}

The proposed system was implemented using the above explained hardware and software under the described flow.

In the system the output is not very accurate. However the output is audible enough and the voice signal can be improved by using the further versions of voice generation module.

In the proposed system, the surrounding noise is major concern. Approximately 15 words have been successfully recognized. The accuracy is only up to $85 \%$ due to the noise and other losses in the system. .The accuracy is further decreased if the user spoken voice tone does not match with the trained voice. We can improve it by using higher versions of the voice control kit but with the increased cost of the system.

\section{CONCLUSION AND FUTURE WORK}

We have presented a novel system which gives synonyms of the words spoken by the user. The system overcomes the real time difficulties of visually impaired people, thereby improving their learning ability. System efficiency is improved with the help of microcontroller 8051 and Voice control kit using IC HM2007. By implementing this system we have realized an interactive learning tool for visually impaired. The current system developed can be made more reliable and flexible. For commercial purposes it can be manufactured at low cost. Also the possible scopes can be as follows:

1. Integrating with Mobile Phone: Using Dictionary as the database the speech processing unit can also be embedded into presently available mobile phones. The dictionary present in the mobile phones can serve as the database for the speech processing unit. As the number of words in the dictionary is usually inexhaustible this could prove to be an efficient and excellent translator.[6]

2. Speech Processing Software: With the advent of high speed processors in present day cell phones, speech processing in real time is not a dream anymore. Thus a monolithic chunk of code could be enough for converting speech to text without preexisting database. This would increase the gamut of the language which needs to be translated.[3]

\section{REFERENCES}

[1] AnuradhaSen, K SamudravijayaSadhana“ Indian accent text to speech system for wed browsing"Vol. 27, Part 1, February 2002, pp. 113-126.

[2] E. Darren Ellis May 2001 "Design of Speaker recognition code using MATLAB" Tennessee 37996

[3] UmezKheradia, AbhaKondwilkar International Journal of Scientific and Research Publications, Volume 2, Issue 12, December 2012 ISSN 2250-3153

[4] Gunashekaran K, Manikandan R. "Sign language to speech translator system using PIC microcontroller" International Journal of Engineering and Technology (IJET) Volume 5 No.2 April-May 2013

[5] C. Duran Ultimate "Trends in Integrated Systems to Enhance Automatic Speech Recognition Performance"

[6] Yong Guan, Lin Zheng, JileiTian Real-time Speaker Adapted Speech to SpeechTranslation System in Mobile Environment

[7] Datasheet of HM2007

[8] Datasheet of SC040

[9] http://www.nskelectronics.com/8051_ver_10.html

[10] http://www.electronics.com/hm2007_speech recognition kit.html 